\title{
First Isolation of Oleate-Dependent Enterococcus faecalis Small-Colony Variants from an Umbilical Exudate of a Pediatric Patient with Omphalitis
}

Noriko Kubota' $^{1)}$, Kei Kuzumoto' ${ }^{1)}$ Eiko Hidaka ${ }^{1)}$, Katsumi Yoshizawa ${ }^{2)}$, Kayoko Yumoto $^{1 \text { \#, Kozue Oana }}{ }^{4,5)}$, Yoshifumi Ogiso ${ }^{1,3)}$, Tomohiko Nakamura ${ }^{3)}$, and Yoshiyuki Kawakami ${ }^{4,5)^{*}}$

Department of Laboratory Medicine ${ }^{1)}$, Department of Surgery 2), Department of Infection Control ${ }^{3)}$, Nagano Children's Hospital, Azumino 399-8288, Japan,

Division of Infection Control and Microbiological Regulation,

Department of Health and Medical Sciences, Shinshu University Graduate School of Medicine ${ }^{4)}$,

Division of Clinical Microbiology,

Department of Biomedical Laboratory Sciences, School of Health Sciences, Shinshu University School of Medicine 5), Matsumoto 390-8621, Japan.

\# Present address; Department of Laboratory Medicine, Nagano Prefectural Suzaka Hospital, Suzaka 382-0091, Japan.

Key Words : oleate-dependent, unsaturated fatty acid, Enterococcus faecalis Running Title: Oleate-dependent $E$. faecalis small-colony variant

Any correspondence on this manuscript should be addressed to:

*Yoshiyuki Kawakami, Ph.D., Professor, Division of Infection Control and Microbiological Regulation, Department of Health and Medical Sciences, Shinshu University Graduate School of Medicine, Matsumoto, 390-8621, Japan.

Phone : +81-263-37-2381; Fax: +81-263-37-2370;

E-mail: yk23724@shinshu-u.ac.jp 
A total number of Figures ----- 5 Figures including 3 Color Figures

A total number of Tables ------ 2 Tables

\section{Summary.}

An oleate-dependent Enterococcus faecalis isolate representing small-colony variants (SCVs) was isolated from the umbilical exudates of a 2-year-7-month-old Japanese male patient in Nagano Children's Hospital, Azumino, Japan. He had been suffering from recurrent omphalitis since his early infancy. The initial E. faecalis SCV isolate demonstrated to form small colonies on sheep blood agar plates and tiny colonies on chocolate and modified Drigalski agar media although no visible growth was observed in thioglycollate broth medium after $48 \mathrm{hrs}$ of incubation in ambient air. We then considered the isolate might possibly be a Streptococcus species. Moreover, the SCV isolate could not be identified by using the MicroScan WalkAway-40 and the API-20-Strep systems, both of which systems yielded the profile numbers not corresponding to any bacterial species, probably caused by the insufficient growth of the isolate in both systems.

However, the SCV isolate was subsequently identified as E. faecalis from the findings of the morphological, cultural, and biochemical properties, and was finally confirmed genetically by sequencing the 16S rRNA gene of the organism. Successive investigation manifested that the addition of oleate, an unsaturated fatty acid, enabled the isolate to grow on every medium with normal-sized colony morphology. Although it has long been well known that long-chain fatty acid especially unsaturated oleic acid should be a major inhibitory agents for growth of a variety of microorganisms including not only mycobacteria but also streptococci, this was, to the best of our knowledge, the first clinical isolation of oleate-dependent E. faecalis SCV isolate. 


\section{Introduction}

In clinical microbiology laboratories, bacterial strains forming small-colony variants (SCVs) which require some kind of compound for growth have been isolated and they have been designated by being given many adjectives, other than SCVs, such as a certain substance-dependent, dwarf, G-variant, and so on. They were demonstrated to represent not only atypical colony morphologies but also to exhibit unusual phenotypic properties (Kahl et al., 2003). A variety of SCVs of gram-positive and gram-negative pathogens have been reported such as Staphylococcus aureus, S. epidermidis, Escherichia coli, Proteus mirabilis, Salmonella enterica, Pseudomonas aeruginosa, and Burkholderia cepacia (Proctor et al., 2006; Oana et al., 2013). The SCV phenotypes are frequently accompanied by genetic mutations that stabilize the SCV phenotypes and often result from hypermutability due to deficient DNA repair (Besier et al., 2008; Schaaff et al., 2003). These mutations often confer resistance to antibiotics and changes in the nutritional requirements such as hemin, menadione, and thymidine dependency (Kahl et al., 1998; Gilligan et al., 1987; von Eiff et al., 1997; Proctor et al., 2006). Among the SCVs, S. aureus SCVs have been intensively studied for their morphological, ultrastructural, metabolic, and growth characteristics (Chatterjee et al., 2007, 2008; Kahl et al., 2003, 2005; Kohler et al., 2003; Proctor, 1998). However, little information has been available concerning the SCVs of Enterococcus species. As far as we know, two cases of endocarditis due to SCVs of E. faecalis (Kaase et al., 2004; Wellinghausen et al., 2009), and only one case of blood-stream infection caused by SCVs of E. faecium occurred in a patient with acute myeloid leukaemia (Gröbner et al., 2012) have been documented to date.

In this report, we present, to the best of our knowledge, the first clinical isolation of oleate-, an unsatulated fatty acid, dependent E. faecalis SCV strain as a causative agent of recurrent omphalitis. Furthermore, characterization of the E. faecalis SCV isolate was carried out concerning phenotypic and biochemical properties together with antimicrobial susceptibilities and growth behaviors. Our microbiological findings concerning the cause of exhibiting SCVs in E. faecalis should bring about the contribution to the deeper understanding of the SCVs of E. faecalis. 
Materials and Methods.

Pathological specimens from a patient yielding Enterococcus faecalis SCVs.

A 2-year-7-month-old Japanese male patient was hospitalized in Nagano Children's Hospital, Azumino, Japan. He had been troubled by persistent umbilical inflammation, and had been diagnosed as suspected urachal remnant. However, careful examination of ultrasound imaging clearly denied the urachal remnant, and disclosed that his diagnosis should be omphalitis. He had received gentamicin ointment chemotherapy in a previous 2 weeks. On the day of his hospitalization, the cotton scrapings from umbilical exudates were submitted to the department of laboratory medicine, Nagano Children's Hospital for bacteriological examinations.

\section{Morphologies and conventional identification of the SCV isolate by biochemical characterization.}

The samples were streaked on chocolate agar (Nippon Becton Dickinson Co., Tokyo, Japan.), sheep blood agar (Trypticase Soy Agar II with 5\% sheep blood, Nippon Becton Dickinson Co.) and modified Drigalski agar (Nippon Becton Dickinson Co.) plates together with inoculating into HK semisolid medium (Kyokuto Seiyaku Co., Tokyo, Japan.) media. After 48 hours of incubation at $35^{\circ} \mathrm{C}$, appearing colonies were morphologically investigated by gram's stain, and subjected to the bacteriological examinations using the Pos Combo 3.1J panels (Sysmex BioMerieux, Kobe, Japan) in the automated microbiological instrument, MicroScan WalkAway system (Siemens Healthcare Diagnostics, Tokyo, Japan), together with API 20 Strep kit system (Nippon bioMérieux, Tokyo, Japan). Catalase activity was performed by observing bubbles after emulsifying a fresh colony in a drop of $5 \% \mathrm{H}_{2} \mathrm{O}_{2}$ on a slide-glass, and ONPG test were carried out using o-nitrophenyl- $\beta$-D-galactopyranoside (Sigma-Aldrich Co., Tokyo, Japan.), according to the procedures described previously (Pezzo, 1994).

\section{Antimicrobial susceptinilities of the SCV isolate.}

Drug susceptibility testings were carried out by the Pos Combo 3.1J panels (Siemens 
Healthcare Diagnostics) in the automated microbiological instrument, MicroScan WalkAway system (Siemens Healthcare Diagnostics). Because of the inability of the SCVs to grow on Mueller-Hinton agar (Nippon Becton Dickinson Co.) plate without blood, susceptibility examinations of the SCVs and the revertants with normal phenotypes were also carried out on Mueller-Hinton agar (Nippon Becton Dickinson Co.) plates with addition of sheep blood using the paper-impregnated discs (Nippon Becton Dickinson Co.). MICs or susceptibilities were determined by gauging the inhibitory diameters appeared around the discs and interpreted according to the CLSI criteria (CLSI).

\section{Molecular identification of the SCV isolate by sequencing the 16S rRNA gene.}

The 16S rRNA gene was directly sequenced as described previously (Neilan et al., 1997) using a DTCS quick start master mix kit (Beckman Coulter Inc., Brea, USA) and a model CEQ2000XL DNA analysis system (Beckman Coulter Inc.). The sequence was retrieved from the Ribosomal Database Project databases (Maidak et al., 2001).

\section{Pulsed-field gel electrophoresis (PFGE) analysis.}

The SCV isolate, the revertant, together with the reference ATCC29212 strains were genotyped by pulsed-field gel electrophoresis (PFGE). The PFGE conditions and the procedures were completely followed according to the protocol as described previously (Oana et al., 2009.) using the restriction enzymes with Sma1 and with Not1, respectively.

\section{Auxotrophy examination of the SCV isolate.}

Auxotrophy testing was performed as follows. Dependency on hemin and NAD was examined using X-factor-, and V-factor-containing XV-Multi disks (Eiken Chemical Co., Tokyo. Japan.) on the Mueller-Hinton (Oxoid) agar plate. Requirement of thymidine, menadion, or biotin were tested by inoculation on the Mueller-Hinton (Oxoid, Basingstoke, U.K.) agar plates containing respective compound (Sigma-Aldrich Co.) at concentrations of $10 \mu \mathrm{g} / \mathrm{ml}$ for menadion, $100 \mu \mathrm{g} / \mathrm{ml}$ for thymidine, and $1000 \mu \mathrm{g} / \mathrm{ml}, 100 \mu \mathrm{g} / \mathrm{ml}, 10 \mu \mathrm{g} / \mathrm{ml}$, and $1 \mu \mathrm{g} / \mathrm{ml}$, respectively, for biotin. Auxotrophy for fatty acids such as oleic acid and lauric acid (Sigma-Aldrich Co.) were examined on the Mueller-Hinton (Eiken Chemical Co.) agar 
plates containing each fatty acid (Sigma-Aldrich Co.) at concentrations of $3.5 \mathrm{mM}$ both for oleic acid and for lauric acid, respectively. The reference Enterococccus faecalis ATCC29212 strain was simultaneously investigated for growth control.

\section{Effect of varying concentrations of fatty acids on the growth of the SCV isolate and reference ATCC29212 strain.}

Mueller-Hinton agar (Eiken Chemical Co.) media were used to determine the effects of long-chain fatty acids on the growth of SCV isolate and reference ATCC29212 strain. The agar media were prepared by adding fatty acids, namely oleic acid and lauric acid to the media at concentrations of $0.035 \mathrm{mM}, 0.35 \mathrm{mM}$, and $3.5 \mathrm{mM}$, respectively. Control medium contained no fatty acid. To further determine the effects of fatty acids on growth, the degrees of growth were determined under the conditions supplemented with a fatty acid by drawing the growth curves using the SCV isolate and the reference ATCC29212 strain. The numbers of viable cells were calculated as CFU per milliliter. At 0, 2, 4, 6, 8, 10, and 12 hrs, the samples $(100 \mu \mathrm{l})$ were removed and 10 -fold serial dilutions were performed. An aliquot $(100 \mu 1)$ of each dilution was spread in duplicate onto sheep blood agar (Trypticase Soy Agar II with 5\% sheep blood, Nippon Becton Dickinson) for colony counts. The inoculated plates were incubated overnight at $35^{\circ} \mathrm{C}$. The growth curves of the SCV isolate obtained under various concentrations of fatty acids were evaluated by comparing them with that of ATCC29212 strain.

\section{Occurrence rate of revertants from the SCVs and SCVs exchanged from the revertants.}

Stability of the isolate with phenotypes of SCVs was assessed to determine the occurrence rate of the revertants with normal-sized colonies from the small to tiny colonies with phenotypes of SCVs. The slightly turbid trypticase-soy broth (Eiken Chemical Co.) culture of the SCVs around logarithmic phase of growth was prepared and the numbers of the cells were counted. An aliquot $(100 \mu l)$ of the properly diluted broth media was spread onto chocolate agar (Nippon Becton Dickinson) plates triplicate. The occurrence rates of revertants were calculated by the number of revertants appeared among the numbers of SCVs. 
As for the frequency of emerging SCVs from revertants, completely the same procedures were also carried out as described above.

\section{Detection of $f a b Z 1$ gene from the SCV isolate and its comparative sequence analysis.}

The gene fabZ1 responsible for the unsaturated fatty acid biosynthase was detected by PCR method using forward (5'-AAA TTG GGA ATT GCA GGC TTT-3') and reverse (5'-TCC AAA ATT GAT TGA TTC TTA TC-3') primers, and was directly sequenced with a DTCS quick start master mix kit and with a CEQ 2000XL DNA analysis system. The sequence was retrieved from the blast databases (Maidak, 2001), and evaluated by comparing it with that of E. faecalis V583 strain.

\section{Results.}

Isolation of the $E$. faecalis SCV isolate and its colonial and morphological properties.

After incubation of the exudates-inoculated media at $35^{\circ} \mathrm{C}$ for $48 \mathrm{hrs}$, as shown in Fig. 1, non-hemolytic small colonies appeared on sheep blood agar (Nippon Becton Dickinson) plate, and tiny colonies on chocolate and modified Drigalski agar (Nippon Becton Dickinson) plates, but no visible growth was observed in HK semisolid medium (Kyokuto Seiyaku Co.). Inoculation of the isolate with small to tiny colonial morphologies onto Mueller-Hinton agar media without any supplementation yielded extremely tiny colonies which might be missing. Among the colonies with phenotypes of SCVs grown on each agar plate, no normal-sized revertant colony was observed, demonstrating uniform growth of small or tiny colonies on respective media.

Appearing SCVs with small or tiny colonial phenotypes were found to be gram-positive, and not uniformly-sized but irregularly-sized cocci as shown in Fig. 2.

\section{Biochemical and molecular identification of the SCV isolate.}

Although direct microscopic examination of the gram-stained umbilical exudate preparation revealed no distinctive pathogens indicative of infection, the SCVs were considered as the causative agent of the infection, because of the repeated isolation of SCVs from specimens submitted two days later. No other microorganisms were detected. 
Biochemical characterization and antimicrobial susceptibilities of the SCVs with negative reaction of catalase activity and with gram-positive coccal shaped morpholology was carried out with the automated microbiological instrument, MicroScan WalkAway system (Siemens Healthcare Diagnostics), together with API 20 Strep kit system (Nippon bioMérieux). The Pos Combo 3.1J panels in the automated microbiological instrument, MicroScan WalkAway system (Siemens Healthcare Diagnostics) could not only correctly identify the isolate, but represented antimicrobial susceptibilities of the isolate. Both of the systems failed to identify the isolate, due to the resulting profile numbers not corresponding to any bacterial species provided with the systems. Indeed, as shown in Table 1, the profile number of 7143110, provided by API 20 Strep system, failed to correspond to any listed profile numbers in the system.

In the next experiment, the $16 \mathrm{~S}$ rRNA gene of the SCV isolate was directly sequenced as described previously (Neilan et al., 1997), and the sequence obtained was retrieved from the Ribosomal Database Project databases (Maidak et al., 2001). Comparative sequence analysis showed complete (100\%) 16S rRNA sequence similarity to that of the type strain of Enterococcus faecalis ATCC19433 (DDBJ / EMBL / GenBank accession no. DQ411814). Therefore, we identified the SCV isolate as E. faecalis.

\section{Auxotrophy examination of the SCV isolate.}

When incubated on the Mueller-Hinton agar media with enrichment of hemin, NAD, thymidine, or menadion, the E. faecalis SCV isolate yielded no growth of normal-sized colonies. Moreover, the E. faecalis SCV isolate was also found to be independent of biotin-requirement. However, in cases of supplementing fatty acids, that is, when incubated on the Mueller-Hinton agar media incorporated with not lauric acid but oleic acid, the SCVs of E. faecalis isolate were manifested to form normal-sized colonies. The finding that the SCV isolate should be dependent on oleic acid was confirmed by adding various concentrations of oleic acid to the agar plates. In consequence, addition of oleic acid at concentrations of $3.5 \mathrm{mM}$ was proved to be adequate for the SCV isolate to yield almost the same-sized colonies as the reference E. faecalis ATCC29212 strain simultaneously used as growth control, as shown in Fig. 3. This was confirmed by drawing the growth curves of 
the SCV isolate by adding various concentrations of oleic acid. The media supplemented with $3.5 \mathrm{mM}$ of oleic acid were demonstrated to support almost the same ample growth as the reference ATCC29212 strain used as growth control, clearly shown in Fig. 4.

\section{Frequency of emergence of normal-sized revertant colonies from the SCV isolate and its} stability.

Normal-sized colonies were demonstrated to emerge among the tiny colonies with the phenotypes of SCVs at frequencies of $5.4 \times 10^{-6}$. In contrast, occurrence of SCVs from normal-sized colonies exchanged from SCVs was extremely low, that is, at frequency of less than $10^{-8}$. We, then, investigated the genetic clonality between the appearing normal-sized colonies and the isolate with SCVs phenotypes by the pulsed-field gel electrophoresis (PFGE) using Not 1 and Sma 1 enzymes and confirmed the clonal identity of the two phenotypes, as shown in Fig. 5.

\section{Demonstration of $f a b Z 1$ gene from the SCV isolate.}

The fabZl gene in E. faecalis SCV isolate, responsible for the biosynthesis of unsaturated fatty acid, was proved to be detected by PCR method and found that the comparative sequence analysis of the fabZ1 gene completely corresponded to each other when compared with that of E. faecalis V583 strain.

\section{Drug susceptibility of the SCV isolate and the revertant strain.}

Both the SCV isolate and the revertant strain exhibited resistances to erythromycin, clindamycin, and also demonstrated high resistance to gentamicin. In addition, the SCV isolate demonstrated the smaller diameters of inhibitory zones to all of the $\beta$-lactam drugs, such as penicillins and cephems, as shown in Table 2, compared with those produced by the revertant strain.

\section{Discussion.}

The specimen of umbilical exudates of a pediatric 2-year-7-month-old Japanese male patient yielded small colonies on sheep blood agar, and tiny colonies on chocolate agar and 
modified Drigalski agar plates, and no visible turbid growth in HK semisolid medium. All of the organisms grown on respective media were catalase-negative and gram-positive cocci with no distinctive chains. No other microorganisms were detected. The appearing colonies reminiscent of streptococci, as shown in Fig. 1, were therefore considered as an agent causing recurrent or persistent omphalitis, and were subjected to the bacteriological examinations. The Pos Combo 3.1J panels (Siemens Healthcare Diagnostics) in the automated microbiological instrument, MicroScan WalkAway system (Siemens Healthcare Diagnostics) could not identify the isolate due to the insufficient growth of the isolate in the system. However, the comparative sequence analysis showed 100\% 16S rRNA sequence similarity to that of the type strain of E. faecalis ATCC19433 ${ }^{\mathrm{TM}}$ (DDBJ/EMBL/GenBank accession no. AE016949). Interestingly, after successive subcultures on sheep blood agar plates, normal phenotypes with usual colonial sizes were encountered among the small colonies with phenotypes of SCVs, as shown in Fig. 1. Subsequent further examination demonstrated the emergence of revertant colonies at frequencies of $5.4 \times 10^{-6}$. The pulsed-field gel electrophoresis (PFGE) analyses of the two phenotypes, that is, the SCV isolate and the revertant strain revealed the same electrophoretic patterns when treated with the endonucleases of Sma 1 as shown in Fig. 5, and Not 1 (data not shown), thus confirming their clonal identities. This fact clearly indicated that normal-sized colonies appeared should be the revertants from the E. faecalis SCV isolate.

Many investigations have investigated concerning the growth inhibitory agents against many bacterial species incorporated in the culture media. Among them, long-chain fatty acids and their derivatives have long been considered as major inhibitory agents for bacterial growth (Pollock, 1947; Carson \& Deneo-Moore, 1980). In fact, the long-chain fatty acids were proved to play inhibitory activities against streptococci (Nieman, 1954), corynebacteria (Cohen et al, 1941; Walker, 1926), lactobacilli (Baunrnfuind, 1942; Strong \& Carpenter, 1942), mycobacteria (Dubos, 1950), and so on. On the other hand, the degree of unsaturation of $\mathrm{C}_{18}$ acids enhanced antimicrobial activity, and increase in carbon chain from $\mathrm{C}_{12}$ to $\mathrm{C}_{18}$ failed to correlate with each other. Moreover, antimicrobial activities of unsaturated and saturated fatty acids were eliminated by addition of bovine serum albumin and/or other compounds (Willett \& Morse, 1966). These findings have been widely 
adopted in preparing many media by incorporating blood, serum, charcoal, and/or so on. In particular, lauric acid $\left(\mathrm{C}_{12}\right)$ was demonstrated to have the stronger inhibitory effect among saturated fatty acids against gram-positive cocci, including E. faecalis. In addition, unsaturated monoenoic acid, such as oleic acid $\left(\mathrm{C}_{18: 1}\right)$ was proved to have stronger inhibitory effect than any saturated fatty acid including lauric acid did (Willett \& Morse, 1966). However, more interesting findings were reported on the dualistic properties depending on the concentrations of fatty acids of palmitoleic, linoleic, and oleic acids, that is, growth stimulatory effect when grown in low concentrations of fatty acid, and growth inhibitory effect when grown in high concentrations of fatty acid (Nieman, 1954).

The role of fatty acids as growth factors for microorganisms has drawn comparatively little attention. However, it has become clear that the fatty acid components of fats can be used in catabolism. This catabolic function of fatty acids were proved, as has not been known well, to be essential in microorganisms (Nieman, 1954). In Escherichia coli strains, the unsaturated fatty acid synthetic pathway have been found to require two specialized proteins, FabA and FabB. However, the FabA and FabB genes are found only in the gram-negative alpha- and gamma-proteobacteria, and as for E. faecalis strains, a protein, annotated as FabZ1, that functionally replaces the E. coli FabA protein, has been demonstrated to be encoded (Wang \& Cronan, 2004), although the sequence of this protein aligns much more closely with E. coli FabZ, a protein that plays no specific role in unsaturated fatty acid synthesis. Therefore, E. faecalis FabZ1 is known to be a bifunctional dehydratase / isomerase, an enzyme activity heretofore confined to a group of gram-negative bacteria. We tried to verify the possession of FabZ1 gene and affirm its existence in the SCV isolate. Nonetheless, the SCV isolate required oleic acid for growth with usual sized colonial morphologies. This fact might imply that the structural gene FabZ1 failed to be expressed by some regulator genes or unknown mechanisms.

On the other hand, the long chain fatty acid such as oleic acid have been known to be deeply related to bacterial respiration and amino acid uptake (Galbraith \& Miller, 1973). In addition, the unsaturated fatty acids were demonstrated to be effected on the $\beta$-galactoside and $\beta$-glucoside transport systems, during the experiment using an unsaturated fatty acid auxotroph of E. coli $\mathrm{K} 12$ grown on the media supplemented with oleic acid or linoleic acid 
(Wilson \& Fox, 1970). In our experiment, as shown in Table 1, the reactions for sorbitol, lactose, and starch were all exchanged from negative in SCV isolate to positive in both revertant and SCV isolate under supplementation with oleic acid. The interesting finding was that the reactions of ONPG ( $\beta$-galactosidase activity) were demonstrated to be positive both for the SCV isolate and the revertant strain. That is, the SCV isolate gave negative reaction for lactose-fermentation in spite of demonstrating positive $\beta$-galactosidase activity. This indicated that the property of being lactose-negative was ascribed not to the absence of $\beta$-galactosidase activity but to the extremely low lactose-permeability of the cell membrane. In short, the oleic acid might actually concerned with the cell membrane permeability of cabohydrates including lactose. That is, the SCV isolate might be considered that they could not catabolize sorbitol, lactose, and starch due to its poor permeability of the cell membrane.

Antimicrobial susceptibility tests by the disc diffusion method revealed, as shown in Table 2, that the SCV isolate was resistant to macrolides and highly resistant to gentamicin and that the inhibitory zone diameters of the SCV isolate against any agents other than gentamicin were also smaller than those of revertant strain, indicating more resistant, which might possibly be explained by the poor permeability of antimicrobials into the cell membrane constructed under oleic acid deficient conditions.

An additional interesting finding was reported that when the Saccharomyces cerevisiae strain was auxotrophic for long-chain fatty acids, this auxotrophic property could be suppressed with high levels of biotin in the medium (Stolz et al., 1999). Moreover, another interesting descriptions (Williams et al., 1947) were noted that in the experiments with lactobacilli, oleic acid was superfluous when biotin was present; they supposed that biotin promotes in some way the formation of unsaturated fatty acids essential to the bacteria. Therefore, since no unsaturated fatty acids would be synthesized in the absence of biotin, they must be added to the medium. Thus, we investigated whether or not the SCV isolate could grow with normal sized colonial morphologies under the circumstances with substantial quantities of biotin, and found that no normal sized colonies appeared even in the biotin-supplemented environment, just as stated in the results section. This experiment 
exhibited that the SCV isolate showing small to tiny colonial morphology should represent true oleate-dependency, having nothing to do with biotin.

To the best of our knowledge, this is the first report of the isolation of oleate-dependent $E$. faecalis SCVs as the etiological agent of a refractory omphalitis occurred in a 2-year-7-month-old Japanese male patient, and our findings are a noteworthy and potentially useful piece of information for deeper understandings of the SCVs of E. faecalis.

\section{Acknowledgments.}

This study was funded by a grant for scientific research from the Ministry of Health and Welfare of Japan.

\section{Conflict of interest.}

The authors have declared that no conflict of interest exists.

\section{References.}

- Baunrnfuind, J. C., Otier, A. L., \& Boruff, C. S. (1942). Growth stimulants in the microbiological assay for riboflavin and pantothenic acid. Ind Eng Chem, Anal Ed, 14, 666-671.

- Besier, S., Zander, J., Kahl, B. C., Kraiczy, P., Brade, V., Wichelhaus, T. A. (2008). The thymidine-dependent small-colony-variant phenotype is associated with hypermutability and antibiotic resistance in clinical Staphylococcus aureus isolates. Antimicrob Agents Chemother, 52, 2183-2189.

- Carson, D.,D., \& Deneo-Moore, L. (1980). Effects of fatty acids on Lysis of Streptococcus faecalis. J Bacteriol 141, 1122-1126.

- Clinical and Laboratory Standards Institute 2008. Performance standards for antimicrobial susceptibility testing, M100-S16, 18th informational supplement. Clinical and Laboratory Standards Institute, Wayne, PA.

- Chatterjee, I., Herrmann, M., Proctor, R. A., Peters, G., \& Kahl., B. C. (2007). Enhanced post-stationary-phase survival of a clinical thymidine-dependent small-colony 
variant of Staphylococcus aureus results from lack of a functional tricarboxylic acid cycle. J Bacteriol 189, 2936-2940.

- Chatterjee, I., Kriegeskorte, A., Fischer, A., Deiwick, S., Theimann, N., Proctor, R.A., Peters, G., Herrmann, M., \& Kahl, B. C. (2008). In vivo mutations of thymidylate synthase (encoded by thy $A$ ) are responsible for thymidine dependency in clinical small-colony variants of Staphylococcus aureus. J Bacteriol 190, 834-842.

- Cohen, S., Snyder, J. C., \& Mueller, J. H. (1941). Factors concerned in the growth of Corynebacterium diphtheriae from minute inocula. J Bacteriol, 41, 581-591.

- DuBos, R. J. (1950). The effect of organic acids on mammalian tubercle bacilli. $J$ Exptl Med, 92, 319-332.

- Galbraith, H. \& Miller, T. B. (1973). Effect of long chain fatty acids on bacterial respiration and amino acid uptake. J Appl Microbiol 36, 659-675.

- Gilligan, P. H., Gage, P. A., Welch, D. F., Muszynski, M. J., Wait, K. R. (1987). Prevalence of thymidine-dependent Staphylococcus aureus in patients with cystic fibrosis. J Clin Microbiol, 25, 1258-1261.

- Gröbner, S., Beck, J., Schaller, M., Autenrieth, I. B., Schulte, B. (2012). Characterization of an Enterococcus faecium small-colony variant isolated from blood culture. Int J Med Microbiol 302, 40-44.

- Kaase, M., Anders, A., \& Gatermann, S. G. (2004). First description of small-colony variants of Enterococcus faecalis isolated from an endocarditis patient. Int J Med Microbiol 294, 146.

- Kahl, B. C., Belling, G., Becker, P., Chatterjee, I., Wardecki, K., Hilgert, K., Cheung, A. L., Peters, G., \& Herrmann, M. (2005). Thymidine-dependent Saphylococcus aureus small-colony variants are associated with extensive alterations in regulator and virulence gene expression profile. Infect Immun 73, 4119-4126.

- Kahl, B. C., Belling, G., Reichelt, R., Herrmann, M., Proctor, R. A., \& Peters G. (2003). Thymidine-dependent small-colony variants of Staphylococcus aureus exhibit gross 
morphological and ultrastructural changes consistent with impaired cell separation. J Clin Microbiol 41, 410-413.

- Kahl, B., Herrmann, M., Everding, A. S., Koch, H. G., Becker, K., Harms, E., Proctor, R. A., Peters, G. (1998). Persistent infection with small colony variant strains of Staphylococcus aureus in patients with cystic fibrosis. J Infect Dis, 177, 1023-1029.

- Kohler, C., von Eiff, C., Peters, G., Proctor, R. A., Hecker, M., \& Engelmann, S. (2003). Physiological characterization of a heme-deficient mutant of Staphylococcus aureus by a proteomic approach. J Bacteriol 185, 6928-6937.

- Maidak, B. L., Cole, J. R., Lilburn, T. G., Parker, C. T. Jr, Saxman, P.R., Farris, R. J., Garrity, G. M., Olsen, G. J., Schmidt, T. M.,\& Tiedje, J. M. (2001). The RDP-II (Ribosomal Database Project). Nucleic Acids Res 29, 173-174.

- Neilan, B. A., Jacobs, D. T., Del Dot, T., Blackall, L. L., Hawkins, P. R., Cox, P. T., \& Goodman, A. E. (1997). rRNA sequences and evolutionary relationships among toxic and nontoxic cyanobacteria of the genus Microcystis. Int J Syst Bacteriol 47, 693-697.

- Nieman, C. (1954). Influence of trace amounts of fatty acids on the growth of microorganisms. Bacteriol Rev 18, 147-163.

- Oana, K., Kawakami, Y., Hayashi, T., \& Ohnishi, M. (2009). Simple broad-spectrum protocol using labiase for bacterial cell lysis to prepare genomic DNA for pulsed-field gel electrophoresis analysis. Microbiol Immmunol 53, 45-48.

- Oana, K., Yamaguchi, M., Nagata, M., Washino, K., Akahane, T., Takamatsu, Y., Tsutsui, C., Matsumoto, T., \& Kawakami, Y. (2013). First isolation of carbon dioxide-dependent Proteus mirabilis from an uncomplicated cystitis patient with Sjögren's syndrome. Jpn J Infect Dis 66, 241-244.

- Pezzo, M. (1995). O-nitrophenyl- $\beta$-D-galactopyranoside test. 1-19-20, Identification of aerobic gram-negative bacteria, section 1. Aerobic bacteriology, In Clinical Microbiology Procedures Handbook, Edited Henry D. Isenberg. ASM Press, Washington, DC.

- Pollock, M., R. (1947). The growth of H. pertussis on media without blood. $B r J$ Exp 
Pathol 28, 295-07.

- Proctor, R. A., Kahl, B. C. von Eiff, P. E. Vaudaux, D. P. Lew, and G. Peters. (1998). Staphylococcal small colony variants have novel mechanisms for antibiotic resistance. Clin Infect Dis 27(Suppl. 1), S68-S74.

- Proctor, R. A., von Eiff, C., Kahl, B. C., Becker, K., McNamara, P., Herrmann, M., \& Peters, G. (2006). Small colony variants: a pathogenic form of bacteria that facilitates persistent and recurrent infections. Nat Rev Microbiol 4, 295-305.

- Schaaff F, Bierbaum G, Baumert N, Bartmann P, Sahl HG. (2003). Mutations are involved in emergence of aminoglycoside-induced small colony variants of Staphylococcus aureus. Int J Med Microbiol, 293, 427-35.

- Stolz, J., Hoja, U., Meier, S., Sauer, N., \& Achweizer, E. (1999). Identification of the plasma membrane $\mathrm{H}^{+}$-biotin symporter of Saccharomyces cerevisiae by rescue of a fatty acid-auxotrophic mutant. J Biol Chem 274, 18743-18746.

- Strong, F. M., \& Carpenter, L. E. (1942). Preparation of samples for microbiological determination of riboflavin. Ind Eng Chem, Anal Ed, 14, 909-913.

- von Eiff, C., Heilmann, C., Proctor, R. A., Woltz, C., Peters, G., Götz, F. (1997). A site-directed Staphylococcus aureus hemB mutant is a small-colony variant which persists intracellularly. J Bacteriol, 179, 4706-4712.

- Walker, J. E. (1926). The germicidal properties of soap. J Infect Dis, 38, 127-130.

- Wang, H., \& Cronan, J. E., (2004). Functional replacement of the FabA and FabB proteins of Escherichia coli fatty acid synthesis by Enterococcus faecalis FabZ and FabF homologues. J Biol Chem 279, 34489-33495.

- Wellinghausen, N., Chatterjee, I., Berger, A., Niederfuehr, A., Proctor, R. A., \& Kahl, B. C. (2009). Characterization of clinical Enterococcus faecalis small-colony variants. $J$ Clin Microbiol 47, 2802-2811.

- Willett, N., P., \& Morse, G., E., (1966). Long-chain fatty acid inhibition of growth of Streptococcus agalactiae in a chemically defined medium. J Bacteriol 91: 2245-2250. 
- Williams, W. L., Broquist, H. P., \& Snell, E. E. (1947). Oleic acid and related compounds as growth factors for lactic acid bacteria. J Biol Chem 170, 619-630.

- Wilson, G., Rose, P., \& Fox, C. F., (1970). The effect of membrane lipid unsaturation on glycoside transport. Biochem Biophys Res Commun 38, 618-623.

Fig. 1

Colony morphologies of $E$. faecalis SCVs and its revertants on various media after $24 \mathrm{~h}$ of incubation

Fig. 2

Gram-stained E. faecalis SCV isolate and its revertant strain compared with that of ATCC29212 strain after incubation for 24h on sheep blood agar plates

Fig. 3

Colonies after 24h of incubation of the SCV isolate grown on Mueller-Hinton agar media supplemented with various concentrations of oleic acid

Fig. 4

Growth curves of the SCV isolate under the conditions supplemented with various concentrations of oleic acid compared with that of ATCC29212 strain without oleic acid

Fig. 5 PFGE of Sma I-digested chromosomal DNAs of E. faecalis SCV isolate and the revertant strain

Lanes 1 and 5, 48.5- to 970-kb molecular weight marker; lane 2, E. faecalis ATCC29212 strain; lane 3, E. faecalis SCV isolate; lane 4, the revertant strain.

Table 1. Several distinct biochemical properties provided by API 20 STREP system

Table 2. Inhibition zone diameters of representative drug-impregnated discs 
Table 1.

Several distinct biochemical properties provided by API 20 STREP system

\begin{tabular}{|c|c|c|c|}
\hline $\begin{array}{l}\text { API } 20 \\
\text { STREP }\end{array}$ & $\begin{array}{l}\mathrm{SCV} \\
\text { isolate }\end{array}$ & $\begin{array}{c}\text { Revertant } \\
\text { strain }\end{array}$ & $\begin{array}{l}\text { SCV isolate under } \\
3.5 \mathrm{mM} \text { oleic acid }\end{array}$ \\
\hline Profile No. & 7143110 & 7143711 & 7143711 \\
\hline Sorbitol & ${ }^{\dagger}$ & $+^{\ddagger}$ & + \\
\hline Lactose & - & + & + \\
\hline Starch & - & + & + \\
\hline ONPG* & + & + & + \\
\hline
\end{tabular}

$\dagger$ - negative reaction $\neq+$ : positive reavtion

* ONPG: o-nitrophenyl- $\beta$-D-galactopyranoside ( $\beta$-galactosidase)

Table 2. Inhibition zone diameters of representative drug-impregnated discs

Antimicrobial Growth inhibition zone diameters $(\mathrm{mm})$ of

\begin{tabular}{lcc}
\multicolumn{1}{c}{ agents } & SCV isolate & Revertant strain \\
\hline Benzylpenicillin & 12.6 & 17.4 \\
\hline Ampicillin & 17.2 & 22.6 \\
\hline Cefazolin & 7.4 & 8.2 \\
\hline Cefotiam & $-*$ & 7.4 \\
\hline Ampicillin/Sulbactam & 12.5 & 18.5 \\
\hline Erythromycin & - & - \\
\hline Clindamycin & - & - \\
\hline Gentamicin & - & - \\
\hline Gentamicin 120\# & - & - \\
\hline Streptomycin 300\# & 14.5 & 14.3 \\
\hline
\end{tabular}

*_- : no formation of inhibitory zone, indicating resistant

\# : containing $120 \mu \mathrm{g}$ and $300 \mu \mathrm{g}$ of drug in each disc 

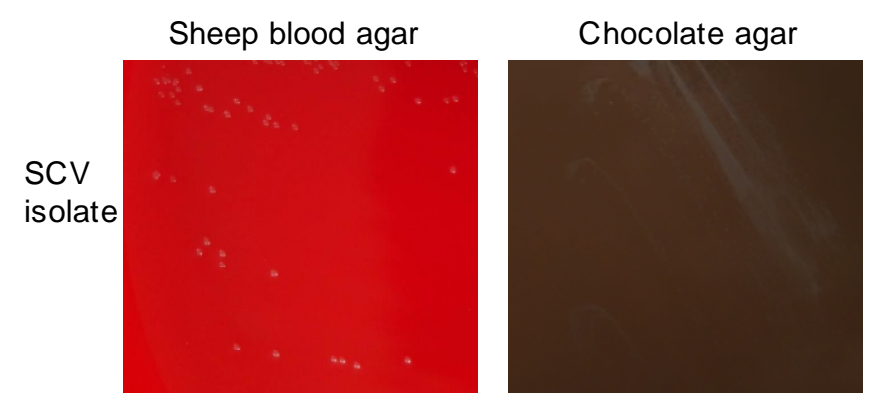

\section{Drigarskiagar}

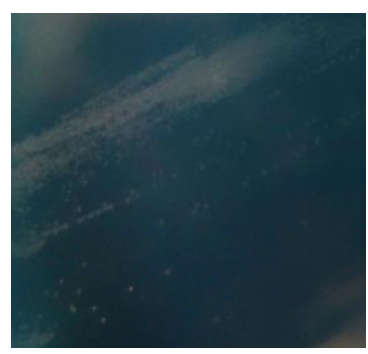

Rev ertant strain
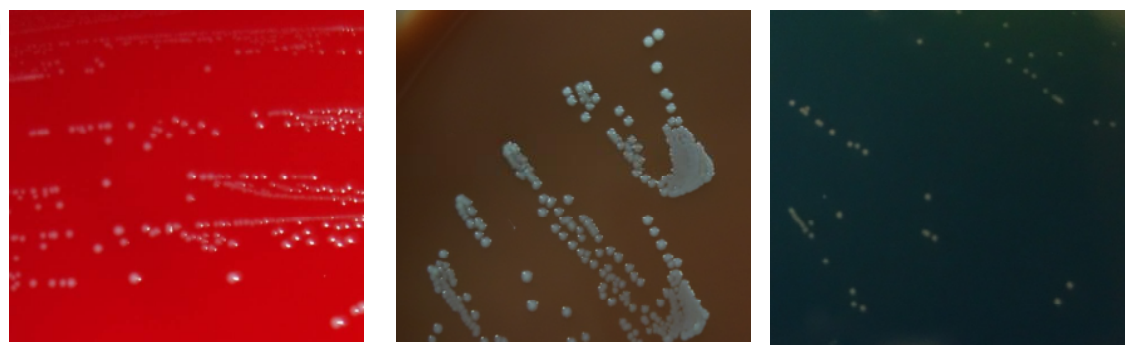

Fig. 1 Colony morphologies 㕵. faecalis SCVs and its rev ertants on $v$ arious media after $24 \mathrm{~h}$ of incubation

\section{ATCC29212 strain}
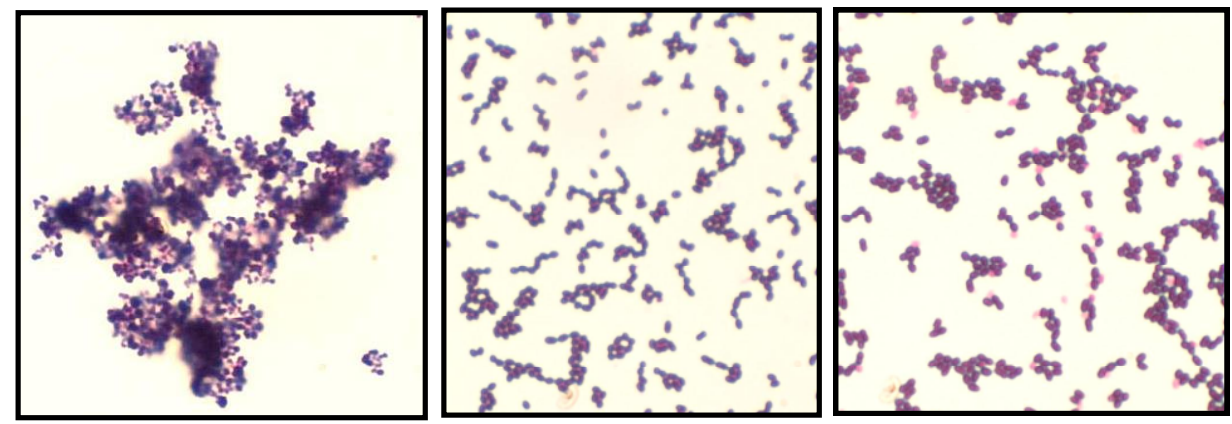
Supplemented with :

$0.035 \mathrm{mM}$ of oleic acid

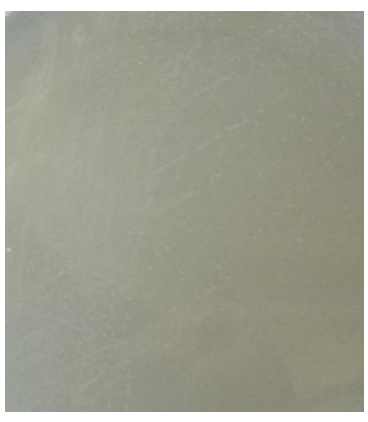

$0.35 \mathrm{mM}$ of oleic acid

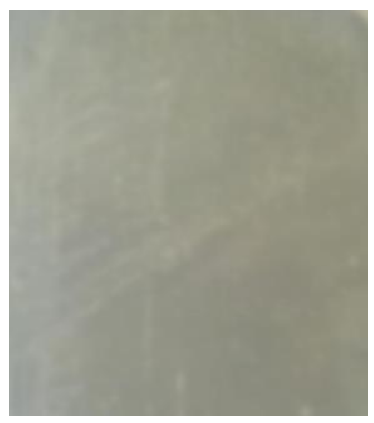

$3.5 \mathrm{mM}$ of oleic acid

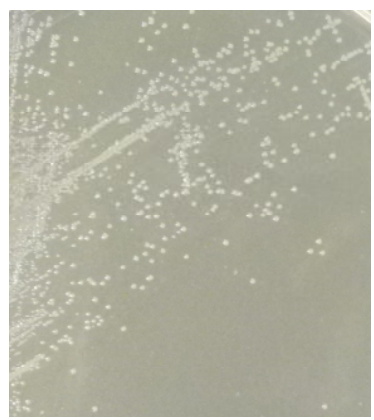

Fig. 3 Colonies after $24 \mathrm{~h}$ of incubation of the SCV isolgtewn on Mueller-Hinton agar media supplemented with various concentrations of oleic acid

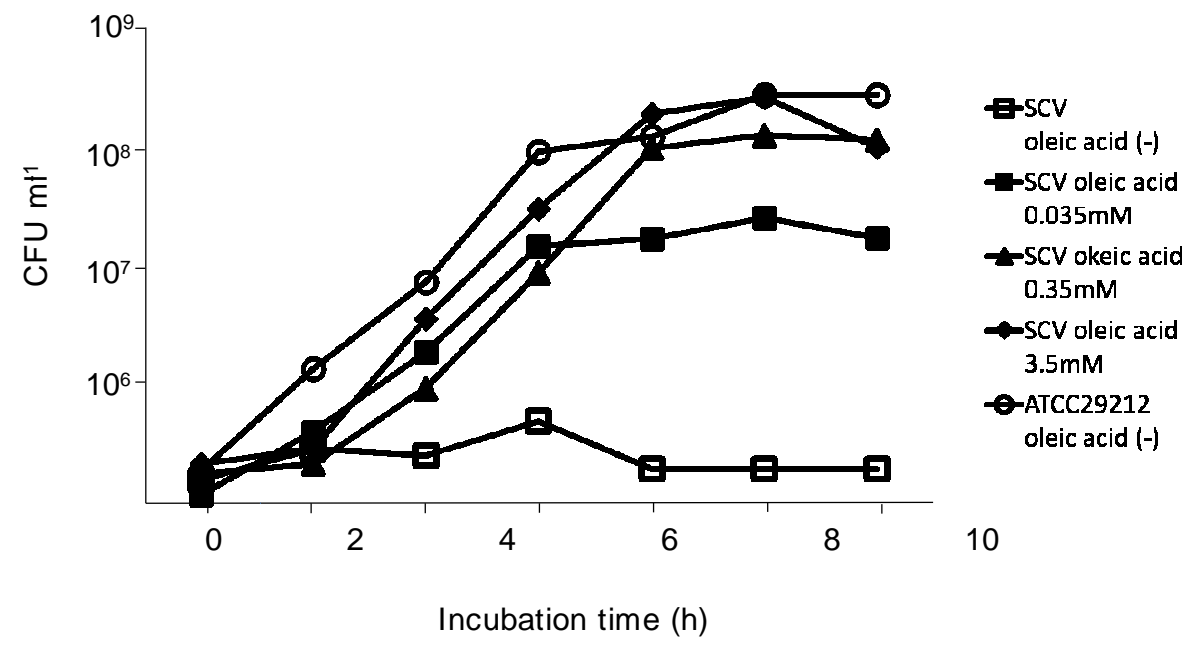

Fig. 4 Growth curves of the SCV isolate under the conditions supplemented with $v$ arious concentrations oleic acid compared with that of ATCC29212 strain without oleic acid 


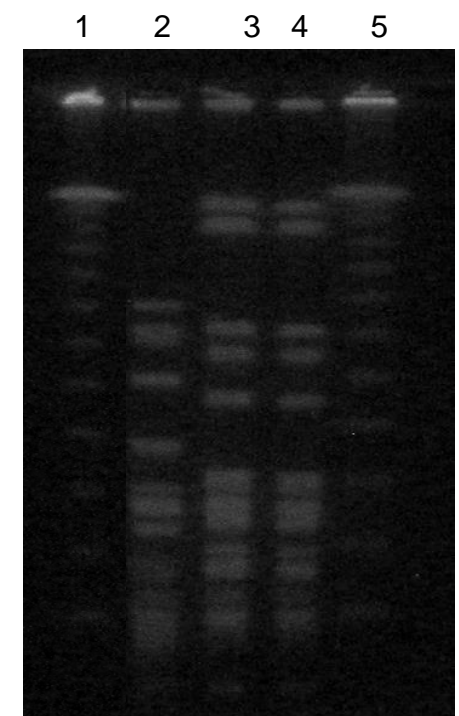

Lanes 1 and 5, 48.5- to 970-kb molecular weight marker; lane $\mathbb{E}$,faecalis ATCC29212 strain; lane 3,E. faecalis SCV isolate; lane 4, therev ertantstrain.

Fig. 5 PFGE of Sma I -digested chromosomalDNAs of E. faecalis SCV isolate andhe rev ertantstrain 